\title{
INFINITE DIVISIBILITY.
}

Mr. Fullerton's extremely ingenious attempt in the October number of MrND to find a new solution to the old puzzles which have come down to us under Zeno's name leads me to think that he and perhaps many others would take some interest in another way of looking at the question.

It is not really an Ontological question. What we are considering is not the true inwardness or real nature of Space or Time, but only our notions or thoughts about them. We want to make our ideas appear consistent with themselves, and when we have done that we shall leave it to the Realist and the Idealist to settle their true meaning if they can.

What we have to do therefore is to avoid all confusion of thought, to distinguish all things which can be distinguished from one another, and with this I think most of the puzzles will disappear. I shall show how we may proceed in the case of space only, because, as Mr. Fullerton remarks, it is a waste of energy to go twice over the same ground.

The first distinction to make is between space and figure. Space is known to us as room to move, but figures are points, lines, surfaces or solids constructed or existing in space. We find space, but we construct figures, and all these puzzles consist in ingenious plans for making us attempt to construct space. Space has no dimensions. Dimension and direction belong to figures. The length of a figure is its size in one direction, but you cannot take the size of space in any direction, nor can you find in space anything to point in one direction rather than another. When we are told that space has three dimensions, an explanation is always given which amounts to what has been just said. We are directed to draw through a point three lines perpendicular to one another, and these we are to take for the three dimensions of space. In point of fact we have constructed a figure and we find that it has three dimensions. The three lines we have drawn explain one another and supply us with three directions in which to measure, but we can in no way attach them to space so as to qualify it.

Again, figures are finite and divisible; space is infinite and indivisible. Take the question of divisibility first. We divide anything when we separate one part of it from the rest. This can only be done by putting between the parts something different from either 
of them. Thus a point will divide a line into twe parts, a line will divide a surface and a surface will divide a solid, and we can divide: a material thing such as a sheet of paper by cutting it in two and. letting some space intervene between the parts, but a line drawn on the paper only divides the surface not the paper, and nothing which we can do on or with the paper in any way affects the space in which it exists. If it were possible to drive two nails into space and stretch a line between them it might be said that space was divided, or if anything which is not space could be inserted between two parts of space then it would be divided indeed, but as nothing of the kind can be done we say that space is indivisible as figure is divisible. And having said that, we have said that space is infinite: but figures are finite, for that is infinite which cannot be increased or diminished, multiplied or divided. Where there is a division there is a boundary, and from what is divided something may betaken away, and to it something may be added, and thus all things. divisible are finite.

In speaking thus, of course by infinity I mean true or Metaphysical infinity. Mathematical infinity is quite another idea and. in its own place an extremely useful one, but we must not allow ourselves to confuse it with true infinity. It is unfortunate that two ideas so different in themselves should have the same name, but. we must take things as we find them, and notwithstanding the difficulty thus caused endeavour to keep our thoughts clear. Mathematical infinity is an endless progression and may be represented as. a line stretching onwards without limit, or a number increasing without end. It is a construction, a sort of tigure, and the line is looked upon as always being drawn onwards, the series of numbers. as always growing, and hence what is Mathematically infinite is Metaphysically finite, since, however far the line may have progressed it may go farther, and however great the number may be it can be made greater.

Now a line is, in the Mathematical sense, infinitely divisible, but is not infinitely divided. To draw a line is to construct a figure; to bisect it when drawn is to construct another figure, but the line has not iwo parts until it has been bisected, or three parts until it has been trisected. If, as Mr. Fullerton suggests, it were necessary to suppose the parts to be there before I make them, it would be equally necessary to suppose the line to exist belore I draw it, and everything else that $I$ do to be done before $I$ do it. The line is capable of having parts, but it has none; it is only one thing until I divide it, and it is capable of being divided without limit, because the process of constructing figures by cutting off smaller and smaller portions is one which gives us no idea as to how it might be made to end.

Mathematicians are in the habit of considering a line to be equivalent to a row of points, and for some purposes this is perfectly satisfactory, but for other purposes it is only an approximation, and 
for Metaphysics it is absolutely false. As a Mathematician, I say that a line is composed of an infinite number of points; as a Metaphysician, I say that a row of points differs from a line as much as a circle does from a triangle. When I am discussing a Metaphysical question, I must keep to the Metaphysical way of looking at things and consider the line to be the continuous course marked out or passed over by a moving body. If the body moved by jerks, the line might be considered to have parts marked out by the beginning of each jerk, but if the motion is continuous, the line is continuoue and not divided, although capable of division, not containing points, although affording a means of distinguishing positions or points. Points, to the Metaphysician, are only positions which must be distinguished or defined by reference to other figures, but which cannot be made to combine because they have no magnitude. Mathematicians add points to points to make lines, and lines to lines to make surfaces, but then the point must be regarded as a small surface obtained by division of a larger surface progressing without limit. From a Mathematician's point of view such a surface differs in no way from a point; to a Metaphysician they are absolutely distinct.

I have called these ideas Metaphysical and Mathematical, but they are evidently part of the furniture of the human mind, easily developed by any one who thinks, but very easily confounded. Zeno's puzzles rest on such confusion, and when it is avoided the difficulty vanishes.

J. N. Shearman. 